\title{
Hartman-Wintner-Type Inequality for a Fractional Boundary Value Problem via a Fractional Derivative with respect to Another Function
}

\author{
Mohamed Jleli, ${ }^{1}$ Mokhtar Kirane, ${ }^{2}$ and Bessem Samet ${ }^{1}$ \\ ${ }^{1}$ Department of Mathematics, College of Science, King Saud University, P.O. Box 2455, Riyadh 11451, Saudi Arabia \\ ${ }^{2}$ LaSIE, Pôle Sciences et Technologies, Université de La Rochelle, avenue M. Crépeau, 17042 La Rochelle Cedex, France \\ Correspondence should be addressed to Bessem Samet; bsamet@ksu.edu.sa
}

Received 2 January 2017; Revised 19 January 2017; Accepted 22 January 2017; Published 12 February 2017

Academic Editor: Thabet Abdeljawad

Copyright (c) 2017 Mohamed Jleli et al. This is an open access article distributed under the Creative Commons Attribution License, which permits unrestricted use, distribution, and reproduction in any medium, provided the original work is properly cited.

\begin{abstract}
We consider a fractional boundary value problem involving a fractional derivative with respect to a certain function $g$. A HartmanWintner-type inequality is obtained for such problem. Next, several Lyapunov-type inequalities are deduced for different choices of the function $g$. Moreover, some applications to eigenvalue problems are presented.
\end{abstract}

\section{Introduction}

In this work, we are concerned with the following fractional boundary value problem:

$$
\begin{aligned}
\left(D_{a, g}^{\alpha} u\right)(t)+q(t) u(t) & =0, \quad a<t<b, \\
u(a) & =u(b)=0,
\end{aligned}
$$

where $(a, b) \in \mathbb{R}^{2}, a<b, \alpha \in(1,2), q:[a, b] \rightarrow \mathbb{R}$ is a continuous function, and $D_{a, g}^{\alpha}$ is the fractional derivative operator of order $\alpha$ with respect to a certain nondecreasing function $g \in C^{1}([a, b] ; \mathbb{R})$ with $g^{\prime}(x)>0$, for all $x \in(a, b)$. A Hartman-Wintner-type inequality is derived for problem (1). As a consequence, several Lyapunov-type inequalities are deduced for different types of fractional derivatives. Next, we end the paper with some applications to eigenvalue problems.

Let us start by describing some historical backgrounds about Lyapunov inequality and some related works. In the late XIX century, the mathematician A. M. Lyapunov established the following result (see [1]).

Theorem 1. If the boundary value problem

$$
\begin{aligned}
u^{\prime \prime}(t)+q(t) u(t) & =0, \quad a<t<b, \\
u(a) & =u(b)=0
\end{aligned}
$$

has a nontrivial solution, where $q:[a, b] \rightarrow \mathbb{R}$ is a continuous function, then

$$
\int_{a}^{b}|q(s)| d s>\frac{4}{b-a} .
$$

Inequality (3) is known as Lyapunov inequality. It is proved to be very useful in various problems in connection with differential equations, including oscillation theory, asymptotic theory, eigenvalue problems, and disconjugacy. For more details, we refer the reader to [2-12] and references therein.

In [8], Hartman and Wintner proved that if boundary value problem (2) has a nontrivial solution, then

$$
\int_{a}^{b}(s-a)(b-s) q^{+}(s) d s>b-a,
$$

where

$$
q^{+}(s)=\max \{q(s), 0\}, \quad s \in[a, b] .
$$

Using the fact that

$$
\max _{a \leq s \leq b}(s-a)(b-s)=\frac{(b-a)^{2}}{4},
$$


Lyapunov inequality (3) follows immediately from inequality (4). Many other generalizations and extensions of inequality (3) exist in the literature; see, for instance, [7, 13-22] and references therein.

Due to the positive impact of fractional calculus on several applied sciences (see, for instance, [23]), several authors investigated Lyapunov-type inequalities for various classes of fractional boundary value problems. The first work in this direction is due to Ferreira [24], where he considered the fractional boundary value problem

$$
\begin{aligned}
\left({ }^{\mathrm{RL}} D_{a}^{\alpha} u\right)(t)+q(t) u(t) & =0, \quad a<t<b, \\
u(a) & =u(b)=0,
\end{aligned}
$$

where $(a, b) \in \mathbb{R}^{2}, a<b, \alpha \in(1,2), q:[a, b] \rightarrow \mathbb{R}$ is a continuous function, and ${ }^{\mathrm{RL}} D_{a}^{\alpha}$ is the Riemann-Liouville fractional derivative operator of order $\alpha$. The main result obtained in [24] is the following fractional version of Theorem 1.

Theorem 2. If fractional boundary value problem (7) has a nontrivial solution, then

$$
\int_{a}^{b}|q(s)| d s>\Gamma(\alpha)\left(\frac{4}{b-a}\right)^{\alpha-1},
$$

where $\Gamma$ is the Gamma function.

Observe that (3) can be deduced from Theorem 2 by passing to the limit as $\alpha \rightarrow 2$ in (8). For other related works, we refer the reader to Ferreira [25, 26], Jleli and Samet [27, 28], Jleli et al. [29, 30], O’Regan and Samet [31], Al Arifi et al. [32], Rong and Bai [33], Chidouh and Torres [34], Agarwal and Özbekler [35], Ma [36], and the references therein.

Very recently, Ma et al. [37] investigated the fractional boundary value problem

$$
\begin{aligned}
\left({ }^{H} D_{1}^{\alpha} u\right)(t)+q(t) u(t) & =0, \quad 1<t<e, \\
u(1) & =u(e)=0,
\end{aligned}
$$

where $\alpha \in(1,2), q:[1, e] \rightarrow \mathbb{R}$ is a continuous function, and ${ }^{H} D_{1}^{\alpha}$ is the Hadamard fractional derivative operator of order $\alpha$. The main result in [37] is the following.

Theorem 3. If fractional boundary value problem (9) has a nontrivial solution, then

$$
\int_{1}^{e}|q(s)| d s>\Gamma(\alpha) \lambda^{1-\alpha}(1-\lambda)^{1-\alpha} e^{\lambda}
$$

where $\lambda=\left(2 \alpha-1-\sqrt{(2 \alpha-2)^{2}+1}\right) / 2$.

In the same paper [37], the authors formulated the following question: How to get the Lyapunov inequality for the following Hadamard fractional boundary value problem:

$$
\begin{aligned}
\left({ }^{H} D_{a}^{\alpha} u\right)(t)+q(t) u(t) & =0, \quad a<t<b, \\
u(a) & =u(b)=0,
\end{aligned}
$$

where $(a, b) \in \mathbb{R}^{2}, 1 \leq a<b, \alpha \in(1,2)$, and $q:[a, b] \rightarrow \mathbb{R}$ is a continuous function. Note that one of our obtained results is an answer to the above question.

\section{Preliminaries}

Before stating and proving the main results in this work, some preliminaries are needed.

Let $I=[a, b]$ be a certain interval in $\mathbb{R}$, where $(a, b) \in$ $\mathbb{R}^{2}, a<b$. We denote by $\mathrm{AC}(I ; \mathbb{R})$ the space of real valued and absolutely continuous functions on $I$. For $n=1,2, \ldots$, we denote by $\operatorname{AC}^{n}(I ; \mathbb{R})$ the space of real valued functions $f(x)$ which have continuous derivatives up to order $n-1$ on $I$ with $f^{(n-1)} \in \mathrm{AC}(I ; \mathbb{R})$; that is,

$$
\begin{aligned}
& \operatorname{AC}^{n}(I ; \mathbb{R})=\left\{f: I \rightarrow \mathbb{R} \text { such that } D^{n-1} f\right. \\
& \left.\quad \in \operatorname{AC}(I ; \mathbb{R})\left(D=\frac{d}{d x}\right)\right\} .
\end{aligned}
$$

Clearly, we have $\operatorname{AC}^{1}(I ; \mathbb{R})=\mathrm{AC}(I ; \mathbb{R})$.

Definition 4 (see [23]). Let $f \in L^{1}((a, b) ; \mathbb{R})$. The RiemannLiouville fractional integral of order $\alpha>0$ of $f$ is defined by

$$
\left({ }^{\mathrm{RL}} I_{a}^{\alpha} f\right)(t)=\frac{1}{\Gamma(\alpha)} \int_{a}^{t} \frac{f(s)}{(t-s)^{1-\alpha}} d s, \quad \text { a.e } t \in[a, b]
$$

Definition 5 (see [23]). Let $\alpha>0$ and $n$ be the smallest integer greater than or equal to $\alpha$. Let $f:[a, b] \rightarrow \mathbb{R}$ be a function such that ${ }^{\mathrm{RL}} I_{a}^{n-\alpha} f \in \mathrm{AC}^{n}([a, b] ; \mathbb{R})$. Then the RiemannLiouville fractional derivative of order $\alpha$ of a function $f$ is defined by

$$
\begin{aligned}
\left({ }^{\mathrm{RL}} D_{a}^{\alpha} f\right)(t) & =\left(\frac{d}{d t}\right)^{n}{ }^{\mathrm{RL}} I_{a}^{n-\alpha} f(t) \\
& =\frac{1}{\Gamma(n-\alpha)}\left(\frac{d}{d t}\right)^{n} \int_{a}^{t} \frac{f(s)}{(t-s)^{\alpha-n+1}} d s
\end{aligned}
$$

for a.e. $t \in[a, b]$.

Let $\alpha>0$ and $n$ be the smallest integer greater than or equal to $\alpha$. By $\operatorname{AC}^{\alpha}([a, b] ; \mathbb{R})$ (see [38]), one denotes the set of all functions $f:[a, b] \rightarrow \mathbb{R}$ that have the representation:

$$
f(t)=\sum_{i=0}^{n-1} \frac{c_{i}}{\Gamma(\alpha-n+1+i)}(t-a)^{\alpha-n+i}+{ }^{\mathrm{RL}} I_{a}^{\alpha} \varphi(t),
$$

$$
\text { a.e } t \in[a, b] \text {, }
$$

where $c_{0}, c_{1}, \ldots, c_{n-1} \in \mathbb{R}$ and $\varphi \in L^{1}((a, b) ; \mathbb{R})$. 
Lemma 6 (see [38]). Let $\alpha>0, n$ be the smallest integer greater than or equal to $\alpha$, and $f \in L^{1}((a, b) ; \mathbb{R})$. Then ${ }^{\mathrm{RL}} D_{a}^{\alpha} f(t)$ exists almost everywhere on $[a, b]$ if and only if $f \in$ $\operatorname{AC}^{\alpha}([a, b] ; \mathbb{R})$; that is, $f$ has representation (15). In such a case, one has

$$
\left({ }^{\mathrm{RL}} D_{a}^{\alpha} f\right)(t)=\varphi(t) \text {, a.e } t \in[a, b] .
$$

Let $g \in C^{1}([a, b] ; \mathbb{R})$ be a nondecreasing function with $g^{\prime}(x)>0$, for all $x \in(a, b)$.

Definition 7 (see [23]). Let $f \in L^{1}((a, b) ; \mathbb{R})$. The fractional integral of order $\alpha>0$ of $f$ with respect to the function $g$ is defined by

$$
\begin{array}{r}
\left(I_{a, g}^{\alpha} f\right)(t)=\frac{1}{\Gamma(\alpha)} \int_{a}^{t} \frac{g^{\prime}(s) f(s)}{(g(t)-g(s))^{1-\alpha}} d s, \\
\text { a.e } t \in[a, b] .
\end{array}
$$

Definition 8 (see [23]). Let $\alpha>0$ and $n$ be the smallest integer greater than or equal to $\alpha$. Let $f:[a, b] \rightarrow \mathbb{R}$ be a function such that $\left(\left(1 / g^{\prime}(t)\right)(d / d t)\right)^{n} I_{a, g}^{n-\alpha} f$ exists almost everywhere on $[a, b]$. In this case, the fractional derivative of order $\alpha$ of $f$ with respect to the function $g$ is defined by

$$
\begin{aligned}
& D_{a, g}^{\alpha} f(t)=\left(\frac{1}{g^{\prime}(t)} \frac{d}{d t}\right)^{n} I_{a, g}^{n-\alpha} f(t) \\
& \quad=\frac{1}{\Gamma(n-\alpha)}\left(\frac{1}{g^{\prime}(t)} \frac{d}{d t}\right)^{n} \int_{a}^{t} \frac{g^{\prime}(s) f(s)}{(g(t)-g(s))^{\alpha-n+1}} d s,
\end{aligned}
$$

for a.e. $t \in[a, b]$. result.

The following lemma is crucial for the proof of our main

Lemma 9. Let $\alpha>0$ and $n$ be the smallest integer greater than or equal to $\alpha$. Suppose that the function $y \mapsto f\left(g^{-1}(y)\right)$ belongs to the space $\mathrm{AC}^{\alpha}([g(a), g(b)] ; \mathbb{R})$. Then

$$
\begin{aligned}
& D_{a, g}^{\alpha} f\left(g^{-1}(y)\right)={ }^{\mathrm{RL}} D_{g(a)}^{\alpha}\left(f \circ g^{-1}\right)(y), \\
& \text { a.e } y \in[g(a), g(b)] .
\end{aligned}
$$

Proof. At first, observe that, from Lemma 6, ${ }^{\mathrm{RL}} D_{g(a)}^{\alpha}(f \circ$ $\left.g^{-1}\right)(y)$ exists for a.e. $y \in[g(a), g(b)]$. Now, using the change of variable $x=g^{-1}(y), y \in(g(a), g(b))$, the chain rule yields

$$
\frac{d}{d y}=\frac{d}{d x} \frac{d x}{d y}=\frac{d}{d x} \frac{1}{g^{\prime}\left(g^{-1}(y)\right)}=\frac{1}{g^{\prime}(x)} \frac{d}{d x} .
$$

Therefore, we obtain

$$
\begin{aligned}
& { }^{\mathrm{RL}} D_{g(a)}^{\alpha}\left(f \circ g^{-1}\right)(y) \\
& =\frac{1}{\Gamma(n-\alpha)}\left(\frac{d}{d y}\right)^{n} \int_{g(a)}^{y} \frac{f\left(g^{-1}(s)\right)}{(y-s)^{\alpha-n+1}} d s \\
& =\frac{1}{\Gamma(n-\alpha)}\left(\frac{1}{g^{\prime}(x)} \frac{d}{d x}\right)^{n} \int_{g(a)}^{g(x)} \frac{f\left(g^{-1}(s)\right)}{(g(x)-s)^{\alpha-n+1}} d s .
\end{aligned}
$$

Next, using the change of variable $t=g^{-1}(s)$, we obtain

$$
\begin{aligned}
& { }^{\mathrm{RL}} D_{g(a)}^{\alpha}\left(f \circ g^{-1}\right)(y) \\
& =\frac{1}{\Gamma(n-\alpha)}\left(\frac{1}{g^{\prime}(x)} \frac{d}{d x}\right)^{n} \int_{a}^{x} \frac{g^{\prime}(t) f(t)}{(g(x)-g(t))^{\alpha-n+1}} d t \\
& =D_{a, g}^{\alpha} f(x)=D_{a, g}^{\alpha} f\left(g^{-1}(y)\right),
\end{aligned}
$$

which proves the desired result.

In the sequel, we denote by $\Xi_{g}([a, b] ; \mathbb{R})$ the functional space defined by

$$
\begin{aligned}
& \Xi_{g}([a, b] ; \mathbb{R})=\left\{f:[a, b] \rightarrow \mathbb{R}: f \circ g^{-1}\right. \\
& \left.\quad \in \mathrm{AC}^{\alpha}([g(a), g(b)] ; \mathbb{R})\right\} .
\end{aligned}
$$

Definition 10 (see [23]). Let $\alpha>0$ and $n$ be the smallest integer greater than or equal to $\alpha$. Let $g(x)=\ln x$, where $x \in[a, b]$ and $0<a<b$. The Hadamard fractional derivative of order $\alpha$ of $f \in \Xi_{g}([a, b] ; \mathbb{R})$ is defined by

$$
{ }^{H} D_{a}^{\alpha} f(t)=D_{a, g}^{\alpha} f(t), \quad \text { a.e } t \in[a, b] .
$$

We refer the reader to Ferreira [24] for the proofs of the following results.

Lemma 11. Let $h \in C([A, B] ; \mathbb{R}),(A, B) \in \mathbb{R}^{2}, A<B$, and $1<\alpha<2$. Then $F \in \operatorname{AC}^{\alpha}([a, b] ; \mathbb{R}) \cap C([a, b] ; \mathbb{R})$ is a solution of the boundary value problem

$$
\begin{gathered}
\left({ }^{\mathrm{RL}} D_{A}^{\alpha} F\right)(t)+h(t)=0, \quad A<t<B, \\
F(A)=F(B)=0
\end{gathered}
$$

if, and only if, $F$ satisfies the integral equation

$$
F(t)=\int_{A}^{B} G(t, s) h(s) d s, \quad A \leq t \leq B,
$$


where

$$
\begin{aligned}
& \Gamma(\alpha) G(t, s) \\
& = \begin{cases}\frac{(t-A)^{\alpha-1}}{(B-A)^{\alpha-1}}(B-s)^{\alpha-1}-(t-s)^{\alpha-1}, & A \leq s \leq t \leq B, \\
\frac{(t-A)^{\alpha-1}}{(B-A)^{\alpha-1}}(B-s)^{\alpha-1}, & A \leq t \leq s \leq B .\end{cases}
\end{aligned}
$$

Lemma 12. The Green function $G$ defined by (27) satisfies the following properties:

(i) $G(t, s) \geq 0$ for all $A \leq t, s \leq B$.

(ii) For all $s \in[A, B]$, one has

$$
\max _{t \in[A, B]} G(t, s)=G(s, s) .
$$

\section{A Hartman-Wintner-Type Inequality for Boundary Value Problem (1)}

In this section, a Hartman-Wintner-type inequality is established for fractional boundary value problem (1).

Problem (1) is investigated under the following assumptions:

(A1) $\alpha \in(1,2)$.

(A2) $q \in C([a, b] ; \mathbb{R})$.

(A3) $g \in C^{1}([a, b]$; $\mathbb{R})$.

(A4) $g$ is a nondecreasing function with $g^{\prime}(x)>0$, for all $x \in(a, b)$.

We have the following result.

Theorem 13. Under assumptions (A1)-(A4), if fractional boundary value problem (1) has a nontrivial solution $u \in$ $\Xi_{g}([a, b] ; \mathbb{R}) \cap C([a, b] ; \mathbb{R})$, then

$$
\begin{aligned}
& \int_{a}^{b}[(g(s)-g(a))(g(b)-g(s))]^{\alpha-1} g^{\prime}(s)|q(s)| d s \\
& \quad \geq \Gamma(\alpha)(g(b)-g(a))^{\alpha-1} .
\end{aligned}
$$

Proof. Suppose that $u$ is a nontrivial solution of (1). Let us define the function $v:[g(a), g(b)] \rightarrow \mathbb{R}$ by

$$
v(y)=u\left(g^{-1}(y)\right), \quad y \in[g(a), g(b)] .
$$

Using Lemma 9, for all $y \in(g(a), g(b))$, we have

$$
\begin{aligned}
D_{a, g}^{\alpha} u\left(g^{-1}(y)\right) & ={ }^{\mathrm{RL}} D_{g(a)}^{\alpha}\left(u \circ g^{-1}\right)(y) \\
& ={ }^{\mathrm{RL}} D_{g(a)}^{\alpha} v(y) .
\end{aligned}
$$

On the other hand, since $u$ is a solution of (1), we have

$$
\begin{aligned}
D_{a, g}^{\alpha} u\left(g^{-1}(y)\right) & =-q\left(g^{-1}(y)\right) u\left(g^{-1}(y)\right), \\
y \in(g(a), g(b)), & \\
u\left(g^{-1}(g(a))\right) & =u\left(g^{-1}(g(b))\right)=0 .
\end{aligned}
$$

Therefore, $v$ is a nontrivial solution of the Riemann-Liouville fractional boundary value problem

$$
\begin{aligned}
\left({ }^{\mathrm{RL}} D_{A}^{\alpha} v\right)(y)+Q(y) v(y) & =0, \quad A<y<B, \\
v(A) & =v(B)=0,
\end{aligned}
$$

where $A=g(a), B=g(b)$, and $Q:[A, B] \rightarrow \mathbb{R}$ is the function defined by

$$
Q(y)=q\left(g^{-1}(y)\right), \quad y \in[A, B] .
$$

Now, by Lemma 11, we obtain

$$
v(y)=\int_{A}^{B} G(y, s) Q(s) v(s) d s, \quad A \leq y \leq B,
$$

where $G$ is the Green function defined by (27). Next, let us consider the Banach space $C([A, B] ; \mathbb{R})$ equipped with the standard norm

$$
\|v\|_{\infty}=\max \{|v(y)|: A \leq y \leq B\} .
$$

Clearly, since $v$ is nontrivial, we have $\|v\|_{\infty}>0$. Further, using (35) and Lemma 12, we have

$$
|v(y)| \leq\|v\|_{\infty} \int_{A}^{B} G(s, s)|Q(s)| d s, \quad y \in[A, B],
$$

which yields

$$
\|v\|_{\infty} \leq\|v\|_{\infty} \int_{A}^{B} G(s, s)|Q(s)| d s .
$$

Therefore, we obtain

$$
\int_{A}^{B} G(s, s)|Q(s)| d s \geq 1 ;
$$

that is,

$$
\int_{g(a)}^{g(b)} G(s, s)\left|q\left(g^{-1}(s)\right)\right| d s \geq 1 .
$$

Using the change of variable $s=g(t)$, we get

$$
\int_{a}^{b} G(g(t), g(t))|q(t)| g^{\prime}(t) d t \geq 1 .
$$

Note that by (27) we have

$$
G(g(t), g(t))=\frac{(g(t)-g(a))^{\alpha-1}(g(b)-g(t))^{\alpha-1}}{\Gamma(\alpha)(g(b)-g(a))^{\alpha-1}},
$$


Therefore,

$$
\begin{aligned}
& \int_{a}^{b}[(g(t)-g(a))(g(b)-g(t))]^{\alpha-1} g^{\prime}(t)|q(t)| d t \\
& \quad \geq \Gamma(\alpha)(g(b)-g(a))^{\alpha-1},
\end{aligned}
$$

which is desired inequality (29).

\section{Lyapunov-Type Inequalities for Different Choices of the Function $g$}

In this section, using Theorem 13, several Lyapunov-type inequalities are deduced for different choices of the function g.

4.1. The Case $g(x)=x^{\beta}, \beta>0$. Taking $g(x)=x^{\beta}, \beta>0$, in Theorem 13, we deduce the following Hartman-Wintner-type inequality.

Corollary 14. If fractional boundary value problem (1) has a nontrivial solution $u \in \Xi_{g}([a, b] ; \mathbb{R}) \cap C([a, b] ; \mathbb{R})$, where $g(x)=x^{\beta}, x \in[a, b], 0<a<b$, then

$$
\begin{gathered}
\int_{a}^{b}\left[\left(s^{\beta}-a^{\beta}\right)\left(b^{\beta}-s^{\beta}\right)\right]^{\alpha-1} s^{\beta-1}|q(s)| d s \\
\quad \geq \frac{\Gamma(\alpha)\left(b^{\beta}-a^{\beta}\right)^{\alpha-1}}{\beta} .
\end{gathered}
$$

Next, let us define the function $\varphi_{\alpha, \beta}:[a, b] \rightarrow[0, \infty)$ by

$$
\varphi_{\alpha, \beta}(s)=\left[\left(s^{\beta}-a^{\beta}\right)\left(b^{\beta}-s^{\beta}\right)\right]^{\alpha-1} s^{\beta-1}, \quad s \in[a, b] .
$$

Since $\varphi_{\alpha, \beta}$ is continuous on $[a, b]$ and $\varphi_{\alpha, \beta}(a)=\varphi_{\alpha, \beta}(b)=0$, there exists some $s^{*}(\alpha, \beta) \in(a, b)$ such that

$$
\varphi_{\alpha, \beta}\left(s^{*}(\alpha, \beta)\right)=\max \left\{\varphi_{\alpha, \beta}(s): s \in[a, b]\right\}>0 .
$$

Therefore, from inequality (44), we obtain the following Lyapunov-type inequality.

Corollary 15. If fractional boundary value problem (1) has a nontrivial solution $u \in \Xi_{g}([a, b] ; \mathbb{R}) \cap C([a, b] ; \mathbb{R})$, where $g(x)=x^{\beta}, x \in[a, b], 0<a<b$, then

$$
\int_{a}^{b}|q(s)| d s \geq \frac{\Gamma(\alpha)\left(b^{\beta}-a^{\beta}\right)^{\alpha-1}}{\beta \varphi_{\alpha, \beta}\left(s^{*}(\alpha, \beta)\right)} .
$$

In order to compute the value of $s^{*}(\alpha, \beta)$ for $\alpha \in(1,2)$ and $\beta>0$, we have to study the variations of the function $\varphi_{\alpha, \beta}$ defined by (45). Observe that

$$
\varphi_{\alpha, \beta}(s)=\phi_{\alpha, \beta}\left(s^{\beta}\right), \quad s \in[a, b],
$$

where $\phi_{\alpha, \beta}:[M, N] \rightarrow[0, \infty)$ is the function defined by

$$
\phi_{\alpha, \beta}(x)=[(x-M)(N-x)]^{\alpha-1} x^{(\beta-1) / \beta},
$$

with $M=a^{\beta}$ and $N=b^{\beta}$. A simple computation yields

$$
\begin{aligned}
& \phi_{\alpha, \beta}^{\prime}(x)=\frac{\phi_{\alpha, \beta}(x)}{x(x-M)(N-x)}\left((\gamma-2 \delta) x^{2}\right. \\
& +(M+N)(\delta-\gamma) x+\gamma M N),
\end{aligned}
$$

for all $x \in(M, N)$, where $\gamma=(1-\beta) / \beta$ and $\delta=\alpha-1$. Next, we put

$$
\begin{array}{r}
P(x)=(\gamma-2 \delta) x^{2}+(M+N)(\delta-\gamma) x+\gamma M N, \\
x \in[M, N] .
\end{array}
$$

We consider three cases.

Case 1 (if $\beta=1 /(2 \alpha-1)$ ). In this case, we have $\gamma=2 \delta$ and $P(x)=0$ if and only if $x=2 M N /(M+N)$. Moreover, we have $P(x) \geq 0$ for $x \in[M, 2 M N /(M+N)]$ and $P(x) \leq 0$ for $x \in[2 M N /(M+N), N]$. Therefore,

$$
\begin{aligned}
\phi_{\alpha, \beta}\left(\frac{2 M N}{M+N}\right) & =\max \left\{\phi_{\alpha, \beta}(x): x \in[M, N]\right\}, \\
s^{*}(\alpha, \beta) & =\left(\frac{2 M N}{M+N}\right)^{1 / \beta}=\left(\frac{2}{a^{\beta}+b^{\beta}}\right)^{1 / \beta} a b .
\end{aligned}
$$

Thus, in this case we obtain

$$
\varphi_{\alpha, \beta}\left(s^{*}(\alpha, \beta)\right)=\left[\frac{\left(b^{\beta}-a^{\beta}\right)^{2}}{4(a b)^{\beta}}\right]^{\alpha-1} .
$$

Next, using (53), we deduce from Corollary 15 the following Lyapunov-type inequality in the case $\beta(2 \alpha-1)=1$.

Corollary 16 (the case $\beta(2 \alpha-1)=1$ ). If fractional boundary value problem (1) has a nontrivial solution $u \in \Xi_{g}([a, b] ; \mathbb{R}) \cap$ $C([a, b] ; \mathbb{R})$, where $g(x)=x^{\beta}, x \in[a, b], 0<a<b$, then

$$
\int_{a}^{b}|q(s)| d s \geq \frac{\Gamma(\alpha)}{\beta}\left[\frac{4(a b)^{\beta}}{b^{\beta}-a^{\beta}}\right]^{\alpha-1} .
$$

Case 2 (if $0<\beta<1 /(2 \alpha-1)$ ). In this case, we have $\gamma>2 \delta>0$ and $P(x)$ has two distinct zeros at

$$
\begin{aligned}
& x_{1}=\frac{(\gamma-\delta)(M+N)-\sqrt{\Delta}}{2(\gamma-2 \delta)}, \\
& x_{2}=\frac{(\gamma-\delta)(M+N)+\sqrt{\Delta}}{2(\gamma-2 \delta)},
\end{aligned}
$$

where

$$
\Delta=(M-N)^{2}(\delta-\gamma)^{2}+4 M N \delta^{2}
$$

It can be easily seen that

$$
0<M<x_{1}<N<x_{2} \text {. }
$$


Moreover, we have $P(x) \geq 0$ for $x \in\left[M, x_{1}\right]$ and $P(x) \leq 0$ for $x \in\left[x_{1}, N\right]$. Therefore,

$$
\begin{aligned}
& \phi_{\alpha, \beta}\left(x_{1}\right)=\max \left\{\phi_{\alpha, \beta}(x): x \in[M, N]\right\}, \\
& s^{*}(\alpha, \beta)=x_{1}^{1 / \beta}=\left(\frac{(1-\alpha \beta)\left(a^{\beta}+b^{\beta}\right)-\sqrt{\left(a^{\beta}-b^{\beta}\right)^{2}(1-\alpha \beta)^{2}+4 a^{\beta} b^{\beta} \beta^{2}(1-\alpha)^{2}}}{2[\beta(1-2 \alpha)+1]}\right)^{1 / \beta} .
\end{aligned}
$$

Case 3 (if $\beta>1 /(2 \alpha-1)$ ). In this case, we have $\gamma<2 \delta$ and $P(x)$ has two distinct zeros at $x_{1}$ and $x_{2}$. It can be easily seen that

$$
x_{2}<M<x_{1}<N
$$

Moreover, we have $P(x) \geq 0$ for $x \in\left[M, x_{1}\right]$ and $P(x) \leq 0$ for $x \in\left[x_{1}, N\right]$. Therefore,

$$
\begin{aligned}
& \phi_{\alpha, \beta}\left(x_{1}\right)=\max \left\{\phi_{\alpha, \beta}(x): x \in[M, N]\right\}, \\
& s^{*}(\alpha, \beta)=x_{1}^{1 / \beta}=\left(\frac{(1-\alpha \beta)\left(a^{\beta}+b^{\beta}\right)-\sqrt{\left(a^{\beta}-b^{\beta}\right)^{2}(1-\alpha \beta)^{2}+4 a^{\beta} b^{\beta} \beta^{2}(1-\alpha)^{2}}}{2[\beta(1-2 \alpha)+1]}\right)^{1 / \beta} .
\end{aligned}
$$

Observe that, for $\beta=1(g(x)=x)$, problem (1) is equivalent to problem (7). Moreover, in this case we have

$$
\begin{gathered}
s^{*}(\alpha, 1)=x_{1}=\frac{a+b}{2} \\
\varphi_{\alpha, 1}\left(s^{*}(\alpha, 1)\right)=\varphi_{\alpha, 1}\left(\frac{a+b}{2}\right)=\left[\frac{(b-a)^{2}}{4}\right]^{\alpha-1} .
\end{gathered}
$$

Therefore, using (61) and Corollary 15, we obtain inequality (8), which is due to Ferreira [24].

4.2. A Lyapunov-Type Inequality via Hadamard Fractional Derivative. Taking $g(x)=\ln x$ in Theorem 13, we deduce the following Hartman-Wintner-type inequality for the following Hadamard fractional boundary value problem:

$$
\begin{aligned}
\left({ }^{H} D_{a}^{\alpha} u\right)(t)+q(t) u(t) & =0, \quad a<t<b, \\
u(a) & =u(b)=0,
\end{aligned}
$$

where $(a, b) \in \mathbb{R}^{2}, 0<a<b, \alpha \in(1,2)$, and $q:[a, b] \rightarrow \mathbb{R}$ is a continuous function.

Corollary 17. If fractional boundary value problem (62) has a nontrivial solution $u \in \Xi_{g}([a, b] ; \mathbb{R}) \cap C([a, b] ; \mathbb{R})$, where $g(x)=\ln x, x \in[a, b]$, then

$$
\int_{a}^{b}\left[\left(\ln \frac{s}{a}\right)\left(\ln \frac{b}{s}\right)\right]^{\alpha-1} \frac{|q(s)|}{s} d s \geq \Gamma(\alpha)\left(\ln \frac{b}{a}\right)^{\alpha-1}
$$

Now, define the function $\psi:[a, b] \rightarrow[0, \infty)$ by

$$
\psi(s)=\left[\left(\ln \frac{s}{a}\right)\left(\ln \frac{b}{s}\right)\right]^{\alpha-1} s^{-1}, \quad s \in[a, b] .
$$

Observe that

$$
\psi(s)=\mu(\ln s), \quad s \in[a, b],
$$

where $\mu:[A, B] \rightarrow[0, \infty)$ is the function defined by

$$
\mu(x)=[(x-A)(B-x)]^{\alpha-1} e^{-x}, \quad x \in[A, B]
$$

with $A=\ln a$ and $B=\ln b$. A simple computation yields

$$
\mu^{\prime}(x)=[(x-A)(B-x)]^{\alpha-2} e^{-x} R(x), \quad x \in(A, B),
$$

where

$$
\begin{aligned}
R(x)= & x^{2}-(2(\alpha-1)+A+B) x+(\alpha-1)(A+B) \\
& +A B, \quad x \in[A, B] .
\end{aligned}
$$

Observe that $R(x)$ has two distinct zeros at

$$
\begin{aligned}
x_{1} & =\frac{2(\alpha-1)+A+B-\sqrt{4(\alpha-1)^{2}+(A-B)^{2}}}{2} \\
& :=\lambda(a, b), \\
x_{2} & =\frac{2(\alpha-1)+A+B+\sqrt{4(\alpha-1)^{2}+(A-B)^{2}}}{2} .
\end{aligned}
$$


It can be easily seen that

$$
A<x_{1}<B<x_{2} .
$$

Moreover, we have $R(x) \geq 0$ for $x \in\left[A, x_{1}\right]$ and $R(x) \leq 0$ for $x \in\left[x_{1}, B\right]$. Therefore, we deduce that

$$
\begin{aligned}
\mu\left(x_{1}\right) & =\max \{\mu(x): x \in[A, B]\}, \\
\psi\left(e^{x_{1}}\right) & =\max \{\psi(s): s \in[a, b]\} \\
& =[(\lambda(a, b)-\ln a)(\ln b-\lambda(a, b))]^{\alpha-1} e^{-\lambda(a, b)} .
\end{aligned}
$$

Next, combining (63) with (72), we obtain the following Lyapunov-type inequality for fractional boundary value problem (62).

Corollary 18. If fractional boundary value problem (62) has a nontrivial solution $u \in \Xi_{g}([a, b] ; \mathbb{R}) \cap C([a, b] ; \mathbb{R})$, where $g(x)=\ln x, x \in[a, b]$, then

$$
\begin{aligned}
& \int_{a}^{b}|q(s)| d s \\
& \quad \geq \Gamma(\alpha)\left[\frac{\ln b-\ln a}{(\lambda(a, b)-\ln a)(\ln b-\lambda(a, b))}\right]^{\alpha-1} e^{\lambda(a, b)},
\end{aligned}
$$

where

$$
\begin{aligned}
& \lambda(a, b) \\
& =\frac{2(\alpha-1)+\ln a+\ln b-\sqrt{4(\alpha-1)^{2}+(\ln a-\ln b)^{2}}}{2} .
\end{aligned}
$$

Observe that, in the particular case $(a, b)=(1, e)$, inequality (73) reduces to inequality (10) which is due to $\mathrm{Ma}$ et al. [37].

Remark 19. Corollary 18 is an answer to the open problem proposed in [37].

\section{Applications to Eigenvalue Problems}

Now, we present an application of the Hartman-Wintner-type inequality given by Theorem 13 to eigenvalue problems.

We say that the scalar $\lambda$ is an eigenvalue of the fractional boundary value problem

$$
\begin{aligned}
\left(D_{a, g}^{\alpha} u\right)(t)+\lambda u(t) & =0, \quad a<t<b, \\
u(a) & =u(b)=0,
\end{aligned}
$$

where $(a, b) \in \mathbb{R}^{2}, a<b, \alpha \in(1,2)$, and $g \in C^{1}([a, b] ; \mathbb{R})$ with $g^{\prime}(x)>0$, for all $x \in(a, b)$, if problem (75) has at least a nontrivial solution $u_{\lambda} \in \Xi_{g}([a, b] ; \mathbb{R}) \cap C([a, b] ; \mathbb{R})$.

We have the following result which provides a lower bound of the eigenvalues of problem (75).

Corollary 20. If $\lambda$ is an eigenvalue of problem (75), then

$$
|\lambda| \geq \frac{\Gamma(\alpha)(B-A)^{\alpha-1}}{\int_{A}^{B}(x-A)^{\alpha-1}(B-x)^{\alpha-1} d x},
$$

where $A=g(a)$ and $B=g(b)$.
Proof. Suppose that $\lambda$ is an eigenvalue of problem (75). Then problem (75) admits a nontrivial solution. Applying Theorem 13 with $q \equiv \lambda$, we obtain

$$
\begin{aligned}
& |\lambda| \int_{a}^{b}(g(s)-g(a))^{\alpha-1}(g(b)-g(s))^{\alpha-1} g^{\prime}(s) d s \\
& \quad \geq \Gamma(\alpha)(g(b)-g(a))^{\alpha-1} .
\end{aligned}
$$

Using the change of variable $x=g(s)$, we obtain

$$
|\lambda| \int_{A}^{B}(x-A)^{\alpha-1}(B-x)^{\alpha-1} d x \geq \Gamma(\alpha)(B-A)^{\alpha-1},
$$

which proves the desired inequality.

Taking $g(x)=x^{\beta}, \beta>0$, in Corollary 20, we obtain the following result.

Corollary 21. If $\lambda$ is an eigenvalue of problem (75) with $g(x)=$ $x^{\beta}, \beta>0, x \in[a, b], 0<a<b$, then

$$
|\lambda| \geq \frac{\Gamma(\alpha)\left(b^{\beta}-a^{\beta}\right)^{\alpha-1}}{\int_{a^{\beta}}^{b^{\beta}}\left(x-a^{\beta}\right)^{\alpha-1}\left(b^{\beta}-x\right)^{\alpha-1} d x} .
$$

Taking $g(x)=\ln x$ in Corollary 20, we obtain the following result.

Corollary 22. If $\lambda$ is an eigenvalue of the Hadamard fractional boundary value problem

$$
\begin{aligned}
\left({ }^{H} D_{a}^{\alpha} u\right)(t)+\lambda u(t) & =0, \quad a<t<b, \\
u(a) & =u(b)=0,
\end{aligned}
$$

where $(a, b) \in \mathbb{R}^{2}, 0<a<b$, and $\alpha \in(1,2)$, then

$$
|\lambda| \geq \frac{\Gamma(\alpha)(\ln b-\ln a)^{\alpha-1}}{\int_{\ln a}^{\ln b}(x-\ln a)^{\alpha-1}(\ln b-x)^{\alpha-1} d x} .
$$

\section{Competing Interests}

The authors declare that there is no conflict of interests regarding the publication of this paper.

\section{Acknowledgments}

The third author extends his appreciation to Distinguished Scientist Fellowship Program (DSFP) at King Saud University (Saudi Arabia).

\section{References}

[1] A. Liapounoff, "Problème général de la stabilité du mouvement," Annales de la faculté des sciences de Toulouse Mathématiques, vol. 9, pp. 203-474, 1907.

[2] A. Beurling, "Un théorème sur les fonctions bornées et uniformément continues sur l'axe réel," Acta Mathematica, vol. 77, pp. 127-136, 1945. 
[3] G. Borg, "On a Liapounoff criterion of stability," American Journal of Mathematics, vol. 71, pp. 67-70, 1949.

[4] R. C. Brown and D. B. Hinton, "Opial's inequality and oscillation of 2nd order equations," Proceedings of the American Mathematical Society, vol. 125, no. 4, pp. 1123-1129, 1997.

[5] R. S. Dahiya and B. Singh, "A Lyapunov inequality and nonoscillation theorem for a second order non-linear differentialdifference equation," Journal of Mathematical and Physical Sciences, vol. 7, pp. 163-170, 1973.

[6] G. S. Guseinov and A. Zafer, "Stability criteria for linear periodic impulsive Hamiltonian systems," Journal of Mathematical Analysis and Applications, vol. 335, no. 2, pp. 1195-1206, 2007.

[7] P. Hartman, Ordinary Differential Equations, John Wiley \& Sons, New York, NY, USA, 1964, Birkhuser, Boston, Mass, USA 1982.

[8] P. Hartman and A. Wintner, "On an oscillation criterion of Liapounoff," American Journal of Mathematics, vol. 73, pp. 885890, 1951.

[9] B. G. Pachpatte, "Inequalities related to the zeros of solutions of certain second order differential equations," Facta Universitatis, Series: Mathematics and Informatics, vol. 16, pp. 35-44, 2001.

[10] W. T. Reid, "A matrix equation related to a non-oscillation criterion and Liapunov stability," Quarterly of Applied Mathematics, vol. 23, pp. 83-87, 1965.

[11] B. Singh, "Forced oscillation in general ordinary differential equations," Tamkang Journal of Mathematics, vol. 6, pp. 5-11, 1975.

[12] A. Wintner, "On the non-existence of conjugate points," American Journal of Mathematics, vol. 73, pp. 368-380, 1951.

[13] D. Çakmak, "Lyapunov-type integral inequalities for certain higher order differential equations," Applied Mathematics and Computation, vol. 216, no. 2, pp. 368-373, 2010.

[14] S. B. Eliason, "Lyapunov type inequalities for certain second order functional differential equations," SIAM Journal on Applied Mathematics, vol. 27, no. 1, pp. 180-199, 1974.

[15] L. Jiang and Z. Zhou, "Lyapunov inequality for linear Hamiltonian systems on time scales," Journal of Mathematical Analysis and Applications, vol. 310, no. 2, pp. 579-593, 2005.

[16] C. Lee, C. Yeh, C. Hong, and R. P. Agarwal, "Lyapunov and Wirtinger inequalities," Applied Mathematics Letters, vol. 17, no. 7, pp. 847-853, 2004.

[17] B. G. Pachpatte, "Lyapunov type integral inequalities for certain differential equations," Georgian Mathematical Journal, vol. 4, no. 2, pp. 139-148, 1997.

[18] S. Panigrahi, "Liapunov-type integral inequalities for certain higher-order differential equations," Electronic Journal of Differential Equations, vol. 28, pp. 1-14, 2009.

[19] N. Parhi and S. Panigrahi, "Liapunov-type inequality for higher order differential equations," Mathematica Slovaca, vol. 52, no. 1, pp. 31-46, 2002.

[20] A. n. Tiryaki, "Recent developments of Lyapunov-type inequalities," Advances in Dynamical Systems and Applications, vol. 5, no. 2, pp. 231-248, 2010.

[21] X. Yang, "On Liapunov-type inequality for certain higher-order differential equations," Applied Mathematics and Computation, vol. 134, no. 2-3, pp. 307-317, 2003.

[22] X. Yang and K. Lo, "Lyapunov-type inequality for a class of evenorder differential equations," Applied Mathematics and Computation, vol. 215, no. 11, pp. 3884-3890, 2010.
[23] A. A. Kilbas, H. M. Srivastava, and J. Trujillo, Theory and Applications of Fractional Differential Equations, vol. 204 of NorthHolland Mathematics Studies, Elsevier Science B.V., Amsterdam, The Netherlands, 2006.

[24] R. A. C. Ferreira, "A Lyapunov-type inequality for a fractional boundary value problem," Fractional Calculus and Applied Analysis, vol. 16, no. 4, pp. 978-984, 2013.

[25] R. A. C. Ferreira, "On a Lyapunov-type inequality and the zeros of a certain Mittag-Leffler function," Journal of Mathematical Analysis and Applications, vol. 412, no. 2, pp. 1058-1063, 2014.

[26] R. A. C. Ferreira, "Lyapunov-type inequalities for some sequential fractional boundary value problems," Advances in Dynamical Systems and Applications, vol. 11, no. 1, pp. 33-43, 2016.

[27] M. Jleli and B. Samet, "Lyapunov-type inequalities for a fractional differential equation with mixed boundary conditions," Mathematical Inequalities \& Applications, vol. 18, no. 2, pp. 443451, 2015.

[28] M. Jleli and B. Samet, "Lyapunov-type inequalities for fractional boundary value problems," Electronic Journal of Differential Equations, vol. 88, pp. 1-11, 2015.

[29] M. Jleli, M. Kirane, and B. Samet, "Lyapunov-type inequalities for fractional partial differential equations," Applied Mathematics Letters, vol. 66, pp. 30-39, 2017.

[30] M. Jleli, L. Ragoub, and B. Samet, "A Lyapunov-type inequality for a fractional differential equation under a Robin boundary condition," Journal of Function Spaces, vol. 2015, Article ID 468536, 5 pages, 2015.

[31] D. O’Regan and B. Samet, "Lyapunov-type inequalities for a class of fractional differential equations," Journal of Inequalities and Applications, vol. 2015, article 247, 10 pages, 2015.

[32] N. Al Arifi, I. Altun, M. Jleli, A. Lashin, and B. Samet, "Lyapunov-type inequalities for a fractional $p$-Laplacian equation," Journal of Inequalities and Applications, vol. 2016, article 189, 2016.

[33] J. Rong and C. Bai, "Lyapunov-type inequality for a fractional differential equation with fractional boundary conditions," Advances in Difference Equations, vol. 2015, article 82, 2015.

[34] A. Chidouh and D. F. Torres, "A generalized Lyapunov's inequality for a fractional boundary value problem," Journal of Computational and Applied Mathematics, vol. 312, pp. 192-197, 2017.

[35] R. P. Agarwal and A. Özbekler, "Lyapunov type inequalities for mixed nonlinear Riemann-Liouville fractional differential equations with a forcing term," Journal of Computational and Applied Mathematics, vol. 314, pp. 69-78, 2017.

[36] D. Ma, "A generalized Lyapunov inequality for a higher-order fractional boundary value problem," Journal of Inequalities and Applications, vol. 2016, article no. 261, 2016.

[37] Q. Ma, C. Ma, and J. Wang, "A Lyapunov-type inequality for a fractional differential equation with Hadamard derivative," Journal of Mathematical Inequalities, vol. 11, no. 1, pp. 135-141, 2007.

[38] D. Idczak and S. Walczak, "Fractional sobolev spaces via Riemann-Liouville derivatives," Journal of Function Spaces and Applications, vol. 2013, Article ID 128043, 15 pages, 2013. 


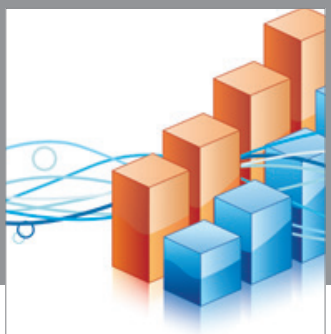

Advances in

Operations Research

vatem alat4

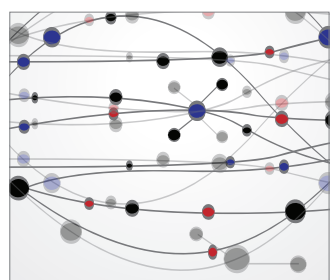

\section{The Scientific} World Journal
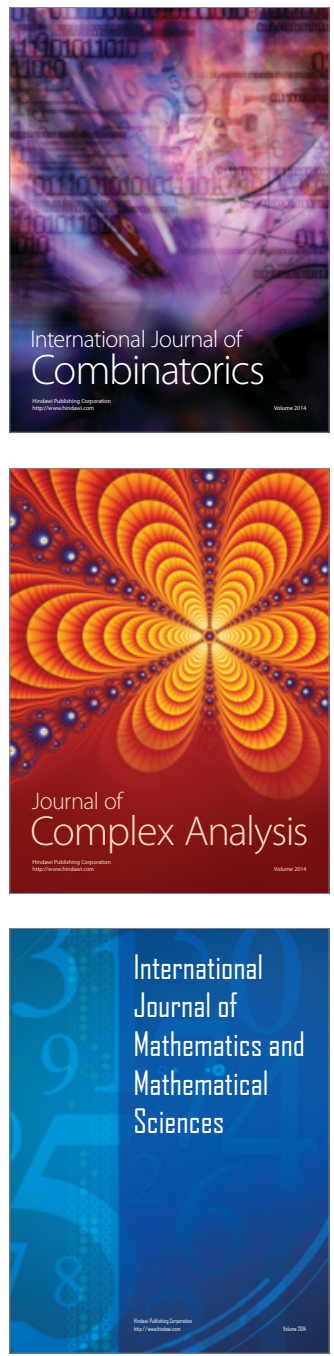
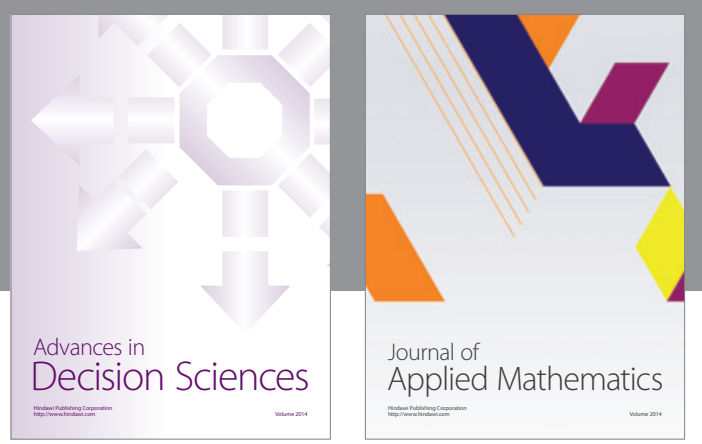

Algebra

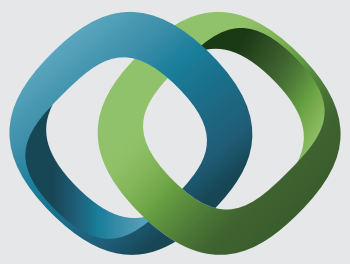

\section{Hindawi}

Submit your manuscripts at

https://www.hindawi.com
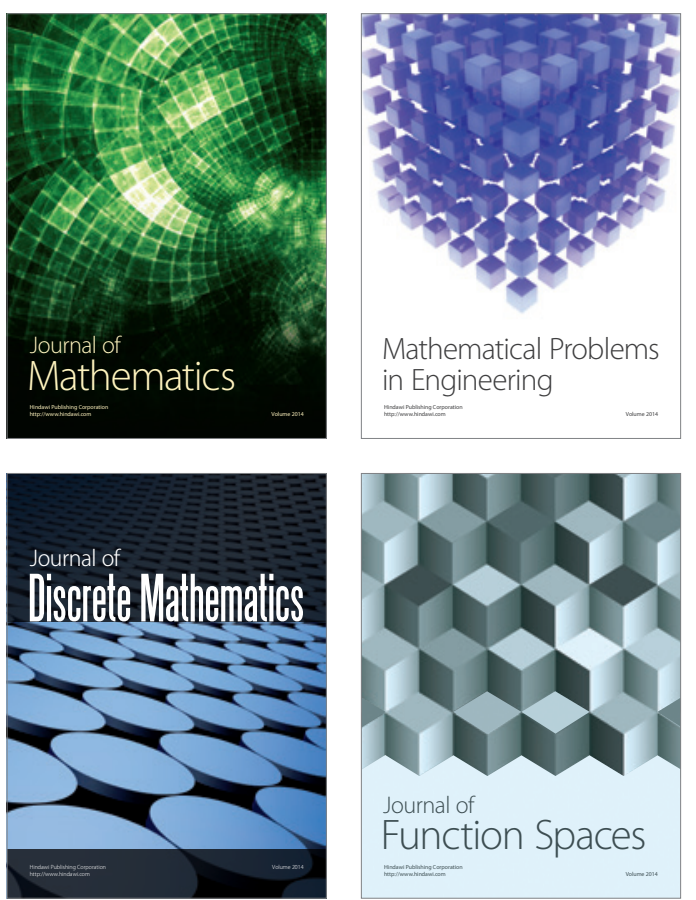

Mathematical Problems in Engineering
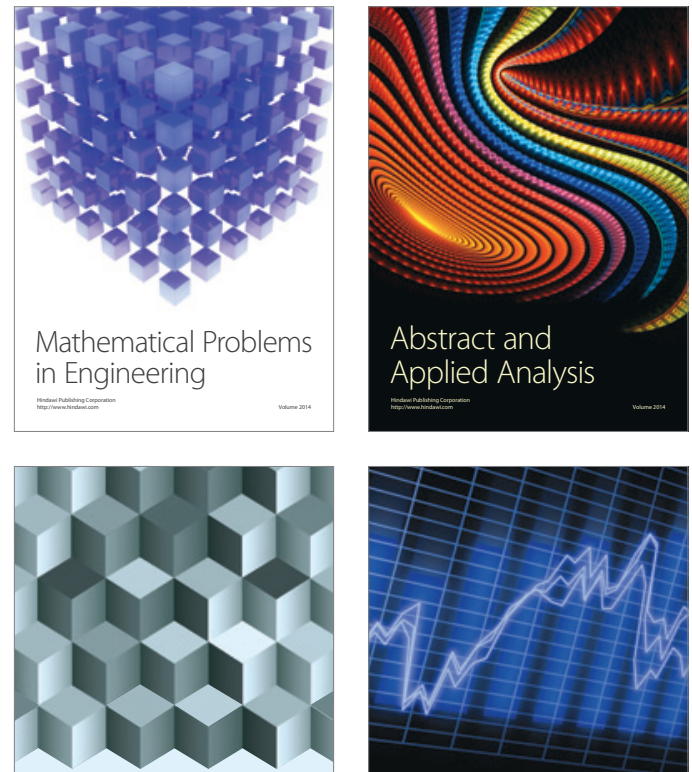

Journal of

Function Spaces

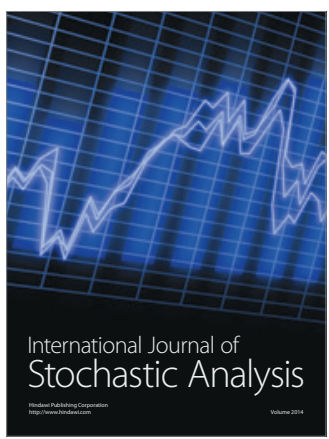

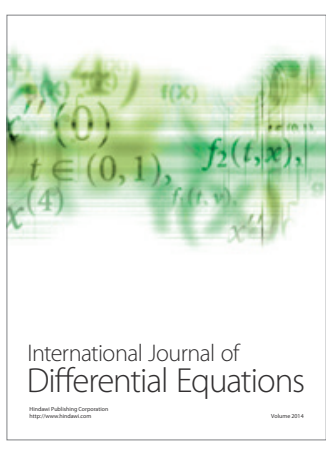
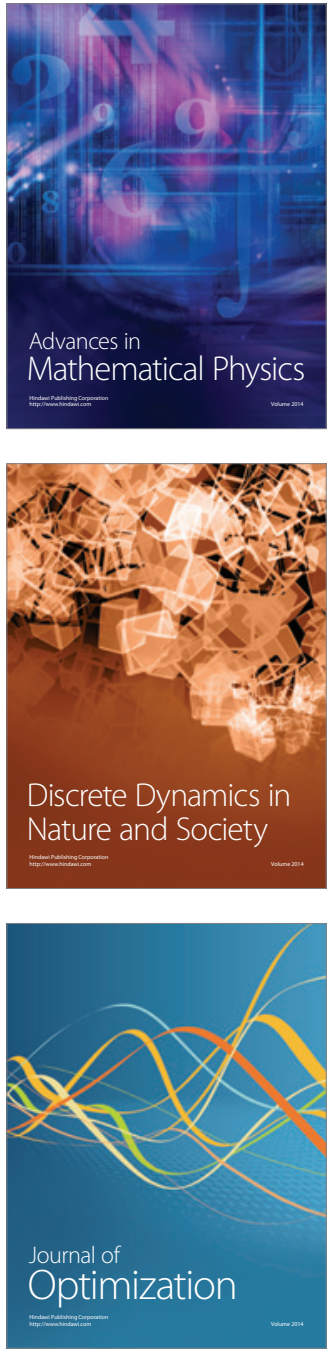